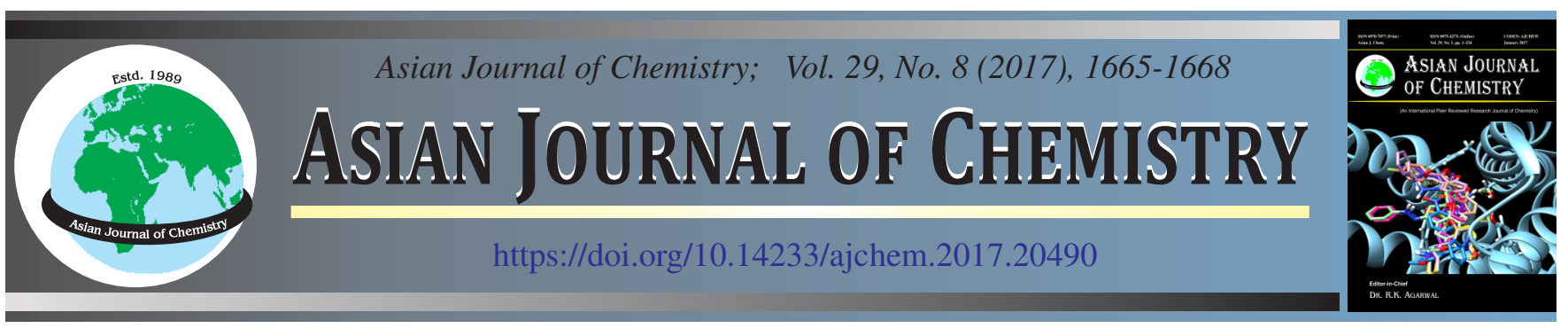

\title{
Synthesis and Characterization of Some New Dioximes from Various Bisaldehydes and their Transformation to Appropriate Diamines
}

\author{
Alireza Banaei ${ }^{*}$, Soheyla Karimi and Maryam SAdeghbari
}

Department of Chemistry, Payame Noor University, Tehran, P.O. Box 19395-4697, Iran

*Corresponding author: Tel: +98 4533515004; E-mail: banaei@pnu.ac.ir

\begin{abstract}
In this work, a series of new dioximes (DASOX) 2(a-c) and diamines 3(a-c) derivatives were successfully synthesized. The bisaldehydes 1a was first prepared by reaction of 5-bromo-2-hydroxy benzaldehyde with 2,3-dibromo propanol in the presence of sodium hydroxide in reflux conditions. The reaction of various bisaldehydes $\mathbf{1}(\mathbf{a}-\mathbf{c})$ with hydroxylamine hydrochloride in an alcoholic solution in the presence of sodium hydroxide results dioximes $\mathbf{2}(\mathbf{a}-\mathbf{c})$ in higher yields. Finally, diamines 3(a-c) was generated by the reduction of dioximes 2(a-c) with $\mathrm{LiAlH}_{4}$ in dry THF. All the newly synthesized compounds characterized by melting point, TLC, elemental analysis, FT-IR, ${ }^{1} \mathrm{H}$ NMR, ${ }^{13} \mathrm{C}$ NMR and Mass spectroscopy.
\end{abstract}

Keywords: Bialdehyde, Dioxime, Hydroxylamine, Diamine.

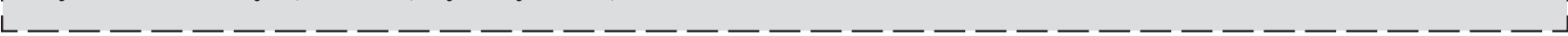

\section{INTRODUCTION}

Oximes are useful intermediates in the organic synthesis, especially for the preparation of amines and reveal certain insecticidal, miticidal and nematocidal activities [1]. Therefore, conversion of carbonyl compounds into oximes is important in synthetic organic chemistry, not only for purification and characterization of carbonyl groups, but also for various functional group transformations which among these are hydrolysis to ketones, reduction to amines or hydroxylamines, oxidation to nitro compounds and for the preparation of amides by the Beckmann rearrangement [2]. Oximes are generally synthesized by condensation of an aldehyde or ketone with hydroxylamine hydrochlorides in the presence of alkali. The reactions of oximes with sodium metal or hydride reagents to give amines, known as reduction to amines are among the most useful and important tools in the synthesis of different kinds of amines [3]. The conversion of carbonyl derivatives to amines via oximes is a useful transformation in the synthesis of numerous organic compounds which are key intermediates in biosynthesis of many pharmacological important substances [4]. Amines derivative have attracted increasing attention due to potential applications in several areas such as biomedicine, biotechnology, commercial drugs and as a starting material for them manufacture of azo dyes. Also, amines are very special species in organic chemistry which exist in many natural biologically important molecules such as amino acids, nucleic acids, alkaloids and many others [5].
In this research paper, we reported the synthesis and characterization of dioximes 2(a-c) from various bisaldehydes $\mathbf{1}(\mathbf{a}-\mathbf{c})$ and transformation of them to appropriate diamines 3(ac). At first, the reaction of 5-bromo-2-hydroxy benzaldehyde with 2,3-dibromo propanol in the presence of sodium hydroxide results in the formation of bisaldehyde (1a). The reaction of bisaldehydes 1a and 2a with hydroxylamine hydrochloride in the presence of sodium hydroxide and ethanol affords dioximes 1a and 2a. Finally, diamines 3(a-c) were synthesized by the reduction of dioximes $\mathbf{2}(\mathbf{a}-\mathbf{c})$ with $\mathrm{LiAlH}_{4}$ in dry THF. The chemical structures of these newly compounds synthesized were characterized by FT-IR, ${ }^{1} \mathrm{H}$ NMR and ${ }^{13} \mathrm{C}$ NMR, Mass spectroscopy and their purities were also confirmed by elemental analyses.

\section{EXPERIMENTAL}

Solvents were dried and purified before being used according to reported procedures [6]. All the reagents were obtained from Merck and used as received, without further purification and most of the solvents were obtained from Rankem. Purity of the compounds is checked by layer chromatography (TLC) using chloroform:ethyl acetate mixture (1:2) as an eluent. All melting points were determined on an Electrothermal 9200 digital melting point apparatus. The IR spectra of the synthesized compounds were recorded on Shimadzu Varian Model4300 FT-IR spectrophotometer in $\mathrm{KBr}$ disc. ${ }^{1} \mathrm{H}$ NMR and ${ }^{13} \mathrm{C}$ NMR spectra were recorded on a Bruker-Avance DRX 400 $\mathrm{MHz}$ spectrometer in $\mathrm{CDCl}_{3}$ and $\mathrm{DMSO}-d_{6}$ solvents using 
TMS as an internal standard. The mass spectra were recorded on a Waters Micromass Q-T of Micro (ESI) spectrometer. Elemental analyses were measured on a Perkin Elmer 240C (CHNO, Rapid) analyzer and agreed with the calculated values.

General Procedure for the synthesis of 6,6'-[ethane1,2-diyile bis(oxy)-1-hydroxymethyl]-bis(3-bromo benzaldehyde) (1a): Bisaldehyde (1a) was prepared by the method of refluxing in alcoholic solution under nitrogen [7]. To a stirred solution of 5-bromo-2-hydroxy benzaldehyde (20.1 g, $0.1 \mathrm{~mol})$ in hot ethanol $(10 \mathrm{~mL})$ was added sodium hydroxide $(4.0 \mathrm{~g}, 0.1 \mathrm{~mol})$ in water $(200 \mathrm{~mL})$. The mixture was warmed and 2,3-dibromo propanol (5.14 $\mathrm{mL}, 0.05 \mathrm{~mol})$ was added. Sufficient ethanol $(150 \mathrm{~mL})$ to produce a homogeneous solution was then added. The progress of reaction was monitored by thin layer chromatography (TLC). The solution was refluxed under nitrogen for $100 \mathrm{~h}$ and then cooled and let stand at $0{ }^{\circ} \mathrm{C}$. The cream coloured crystals produced were washed with water. Recrytallization was performed using an ethanolwater mixture. The resulting crystals were collected by filtration and dried in vacuum desiccators over $\mathrm{P}_{4} \mathrm{O}_{10}$.

Yield $38 \%$; m.p.: $140{ }^{\circ} \mathrm{C}$. IR $\left(\mathrm{KBr}, \mathrm{v}_{\max }, \mathrm{cm}^{-1}\right): 3400(\mathrm{OH})$, $1670(\mathrm{C}=\mathrm{O}), 1242(\mathrm{C}-\mathrm{O}) ;{ }^{1} \mathrm{H}$ NMR $\left(\mathrm{CDCl}_{3}, 400 \mathrm{MHz}\right): \delta$ : 10.33 (s, 2H), 7.91 (d, 2H), 7.66 (d-d, 2H), 6.96 (d, 2H), 4.53 $(\mathrm{m} 1 \mathrm{H}), 4.31(\mathrm{dd}, 4 \mathrm{H}), 2.86(\mathrm{~s}, 1 \mathrm{H}) \mathrm{ppm} ;{ }^{13} \mathrm{C} \mathrm{NMR}\left(\mathrm{CDCl}_{3}\right.$, $400 \mathrm{MHz})$ : $\delta$ : $68.271\left(\mathrm{CH}_{2} \mathrm{OH}, \mathrm{C}-1\right), 69.672\left(\mathrm{OCH}_{2}, \mathrm{C}-3\right), 77$ (CH, C-2), 114.340 (C-Br, C-7), 115.049 (CH, C-5), 126.372 (C, C-9), 132.619 (CH, C-8), 138.469 (CH, C-6), 159.122 (C, C-4), 188.067 (CH, C-10) ppm; Ms: $m / z=458(\mathrm{M})^{+}$. Anal. calcd. (\%) for $\mathrm{C}_{17} \mathrm{H}_{14} \mathrm{O}_{5} \mathrm{Br}_{2}$ : C 81.01, H 5.48; Found (\%): C 81.07, $\mathrm{H}$ $5.42 \%$.

Bisaldehydes 2,2'-[ethane-1,2-diyile bis(oxy)-1-methyl hydroxy]benzaldehyde (1b) and 2,2'-[butane-1,4-diyile bis(oxy)]bis(3-methoxybenzaldehyde) (1c) were prepared according to a reported method in literature [7].

Synthesis of deoximes 2-(a-c): To stirred solution of bisaldehydes $\mathbf{1}(\mathbf{a}-\mathbf{c})(2.29 \mathrm{~g}, 5 \mathrm{mmol})$ in ethanol $(150 \mathrm{~mL})$ was added mixture of hydroxylamine hydrochloride $(3.47 \mathrm{~g}, 50$ mmol) and sodium hydroxide $(2.4 \mathrm{~g}, 60 \mathrm{mmol})$ in $70 \mathrm{~mL}$ water. The solution was refluxed for $24 \mathrm{~h}$ and then added $100 \mathrm{~mL}$ $\mathrm{HCl}(2 \mathrm{M})$ to give precipitate. The progress of reaction was monitored by thin layer chromatography (TLC). The crystals produced were filtered and washed with water. Recrytallization was performed using an ethanol-water mixture. The resulting crystals were collected by filtration and dried in vacuum desiccators over $\mathrm{P}_{4} \mathrm{O}_{10}$.

5-Bromo-2-[2-(4-bromo-2-(hydroxy imino methyl) fenoxy) ethoxy] benzaldehy-deoxime (2a): Yield $84 \%$; m.p.; $155^{\circ} \mathrm{C}$. IR $\left(\mathrm{KBr}, v_{\max }, \mathrm{cm}^{-1}\right): 3400(\mathrm{OH}), 1635(\mathrm{C}=\mathrm{N}), 1250$ (C-O); ${ }^{1} \mathrm{H}$ NMR (DMSO, $\left.400 \mathrm{MHz}\right): \delta: 11.45$ (s, 2H, NOH), 8.34 (s, 2H, H-9), 7.75 (d, 2H, H-8), 7.5 (d-d, 2H, H-6), 7.1 (d, 2H, H-5), 5.6 (s, 1H, OH), 4.2 (m, 1H, H-2), 4.15 (d-d, 2H, $\mathrm{H}-3$ ), 4.1 (dd, 2H, H-1) ppm; ${ }^{13} \mathrm{C}$ NMR (DMSO, $400 \mathrm{MHz}$ ):

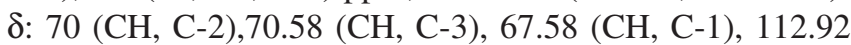
(C, C-7), 115.71 (CH, C-5), 124.01 (C, C-9), 127.78 (CH, C8), 133.38 (CH, C-6), 142.95 (CH, C-10), 155.75 (C, C-4) ppm; Ms: $m / z=488(\mathrm{M})^{+}$. Anal. calcd. $(\%)$ for $\mathrm{C}_{17} \mathrm{H}_{16} \mathrm{~N}_{2} \mathrm{O}_{5} \mathrm{Br}_{2}$ : C 79.68, H 6.18, N 11.95; Found (\%): C 79.57, H 6.23 N $11.68 \%$.
2-[2-(2-(Hydroxyl imino methyl)fenoxy)ethoxy]benzaldehydeoxime (2b): Yield $60 \%$; m.p.; $172{ }^{\circ} \mathrm{C}$. IR (KBr, $\mathrm{v}_{\max }$, $\left.\mathrm{cm}^{-1}\right)$ : $3400(\mathrm{OH}), 1630(\mathrm{C}=\mathrm{N}), 1260(\mathrm{C}-\mathrm{O}) ;{ }^{1} \mathrm{H}$ NMR (DMSO, $400 \mathrm{MHz}$ ): $\delta: 11.19$ (s, 2H, NOH), 8.41 (s, 2H, H-9), 7.66 (d, 2H, H-8), 7.36 (dd, 2H, H-6), 7.10 (d, 2H, H-5), 5.5 (s, 1H, $\mathrm{OH}), 4.2$ (m, 1H, H-2), 4.17 (d-d, 2H, H-3,1), 6.98 (t, 2H, H7) ppm; ${ }^{13} \mathrm{C}$ NMR (DMSO, $\left.400 \mathrm{MHz}\right): \delta: 70(\mathrm{CH}, \mathrm{C}-2), 69.75$ (CH, C-3), 67.44 (CH, C-1), 120.84 (C, C-7), 112.72 (CH, C5), 121.34 (C, C-9), 125.36 (CH, C-8), 130.74 (CH, C-6), $143.64(\mathrm{CH}, \mathrm{C}-10), 158.13$ (C, C-4) ppm; Ms: $m / z=330(\mathrm{M})^{+}$. Anal. calcd. (\%) for $\mathrm{C}_{17} \mathrm{H}_{18} \mathrm{~N}_{2} \mathrm{O}_{5}$ : C 74.34, H 6.42, N 11.28; Found (\%): C 74.13, H 6.48, N $11.34 \%$.

2-[4-(2-(Hydroxy imino methyl)-6-methoxyfenoxy)butoxy]-3-methoxybenzaldehydeoxime (2c): Yield $98 \%$; m.p.; $155^{\circ} \mathrm{C}$. IR (KBr, $\left.v_{\max }, \mathrm{cm}^{-1}\right)$ : $1635(\mathrm{C}=\mathrm{N}), 1269$ (C-O); ${ }^{1} \mathrm{H}$ NMR (DMSO, $\left.400 \mathrm{MHz}\right): \delta: 11.32$ (s, 2H, NOH), 8.41 (s, 2H, H-9), 3.81 (s, 6H, H-12), 8.28 (s, 2H, H-11), 7.28 (t, 2H, H8), 5.5 (s, 1H, OH), 7.06 (d, 4H, H-7,9), 4.17 (t, 4H, $\mathrm{OCH}_{2} \mathrm{CH}_{2}$ ), 1.87 (t, 4H, H-2,3) ppm; ${ }^{13} \mathrm{C} \mathrm{NMR} \mathrm{(DMSO,} 400 \mathrm{MHz}$ ): $\delta$ : $56.17\left(\mathrm{OCH}_{3}, \mathrm{C}-12\right), 146.51(\mathrm{CH}, \mathrm{C}-11), 117.31$ (C, C-10), 126.97 (CH, C-9), 124.59 (CH, C-8), 113.97 (CH, C-7), 153.16 (C, C-6), 143.96 (C, C-5), $26.69\left(\mathrm{CH}_{2}, \mathrm{OCH}_{2} \mathrm{CH}_{2}\right), 73.45\left(\mathrm{OCH}_{2}\right.$, $\mathrm{OCH}_{2} \mathrm{CH}_{2}$ ) ppm; Ms: $m / z=388(\mathrm{M})^{+}$. Anal. calcd. $(\%)$ for $\mathrm{C}_{20} \mathrm{H}_{24} \mathrm{~N}_{2} \mathrm{O}_{6}$ : C 81.14, H 7.09, N 10.59; Found (\%): C 81.07, H 7.17, N-10.56\%.

Synthesis of diamines 3(a-c): To a stirred solution of dioximes 2(a-c) (1.95 g, 4 mmol) in dry THF (150 mL) was added lithium aluminum hydride $(0.91 \mathrm{~g}, 24 \mathrm{mmol})$ and remained in $0{ }^{\circ} \mathrm{C}$ for $0.5 \mathrm{~h}$. The solution was refluxed for $24 \mathrm{~h}$ and then cooled it and added water to remove Li. This reaction should be carried out under nitrogen. The progress of reaction was monitored by thin layer chromatography (TLC). The product was extracted by dichloromethane and $100 \mathrm{~mL} \mathrm{NaOH}(1 \mathrm{M})$.

2,3-Bis[2-amino methyl)-4-bromo fenoxy]propane-1ole (3a): Yield $75 \%$; IR (KBr, $\left.\nu_{\max }, \mathrm{cm}^{-1}\right): 3400(\mathrm{~N}-\mathrm{H}), 3400$ (OH), 1244 (C-O), 754 (C-Br); ${ }^{1} \mathrm{H}$ NMR $\left(\mathrm{CDCl}_{3}, 400 \mathrm{MHz}\right)$ :

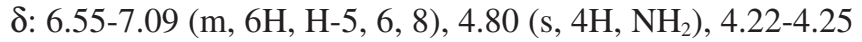
(d-d, 4H, H-1, 3), 4.04-4.14 (m, 1H, H-2), 3.92 (s, 4H, H-10), 2.78 (s, $1 \mathrm{H}, \mathrm{OH}) \mathrm{ppm} ;{ }^{13} \mathrm{C} \mathrm{NMR}\left(\mathrm{CDCl}_{3}, 400 \mathrm{MHz}\right): \delta: 81$ (CH, C-2),73.6 (CH, C-3), 64.3 (CH, C-1), 114.5 (C, C-7), 116.2 (CH, C-5), 130.3 (C, C-9), 131 (CH, C-8), 130.-4 (CH, C-6), 37.2 (CH, C-10), 156.4 (C, C-4) ppm; Ms: $\mathrm{m} / \mathrm{z}=460$ (M) ${ }^{+}$. Anal. calcd. (\%) for $\mathrm{C}_{17} \mathrm{H}_{20} \mathrm{~N}_{2} \mathrm{O}_{3} \mathrm{Br}_{2}$ : C 71.53, H 6.01, N 10.38; Found (\%): C 71.41, H 6.07 N $10.32 \%$.

2,3-Bis[2-amino methyl)fenoxy]propane-1-ole (3b): Yield $78 \%$; IR (KBr, $\left.v_{\max }, \mathrm{cm}^{-1}\right): 3400(\mathrm{~N}-\mathrm{H}), 3400(\mathrm{OH})$, 1240 (C-O); ${ }^{1} \mathrm{H}$ NMR $\left(\mathrm{CDCl}_{3}, 400 \mathrm{MHz}\right): \delta$ : 6.69-7.18 (m, $8 \mathrm{H}, \mathrm{H}-5,6,7,8), 4.75$ (s, 4H, NH $\mathrm{N}_{2}$, 4.04-4.07 (d-d, 2H, H-1), 4.10-4.13 (d-d, 2H, H-3), 4.17-4.24 (m, 1H, H-2); 3.75 (s, $4 \mathrm{H}, \mathrm{H}-10) ; 2.7$ (s, 1H, OH) ppm; ${ }^{13} \mathrm{C} \mathrm{NMR}\left(\mathrm{CDCl}_{3}, 400 \mathrm{MHz}\right)$ :

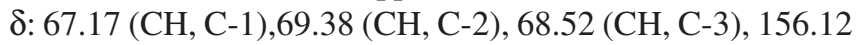
(C, C-4), 111.78 (CH, C-5), 127.85 (CH, C-6), $120.18(\mathrm{CH}$, C-7), 128.-50 (CH, C-8), 128.95 (C, C-9), -41.06 (CH, C-10) ppm; Ms: $m / z=302(\mathrm{M})^{+}$. Anal. calcd. $(\%)$ for $\mathrm{C}_{17} \mathrm{H}_{22} \mathrm{~N}_{2} \mathrm{O}_{3}: \mathrm{C}$ 68.87, H 7.18, N 10.39; Found (\%): C 68.79, H 7.23, N $10.31 \%$.

[Butane 1,4-diyile bis(oxy)]bis(3-methoxy1,2-phenylene)dimethanamine (3c): Yield $70 \%$; IR $\left(\mathrm{KBr}, v_{\max }, \mathrm{cm}^{-1}\right)$ : 
3380 (N-H), 1271 (C-O); ${ }^{1} \mathrm{H}$ NMR $\left(\mathrm{CDCl}_{3}, 400 \mathrm{MHz}\right): \delta: 1.75$ (t, $\left.4 \mathrm{H}, \mathrm{OCH}_{2} \mathrm{CH}_{2}\right), 4.08$ (t, $\left.4 \mathrm{H}, \mathrm{OCH}_{2} \mathrm{CH}_{2}\right), 4.75$ (s, $4 \mathrm{H}, \mathrm{NH}_{2}$ ), -6.82-6.89 (d-, 4H, H-7, 9), 7-7.05 (t, 2H, H-8), 2.01 (s, 4H, $\mathrm{H}-11) ; 3.85$ (s, 6H, H-12); 2.7 (s, 1H, OH) ppm; ${ }^{13} \mathrm{C}$ NMR $\left(\mathrm{CDCl}_{3}, 400 \mathrm{MHz}\right): \delta$ : $72.82\left(\mathrm{OCH}_{2}, \mathrm{OCH}_{2} \mathrm{CH}_{2}\right), 27.10\left(\mathrm{CH}_{2}\right.$, $\left.\mathrm{OCH}_{2} \mathrm{CH}_{2}\right), 146.06$ (C, C-5), 152.70 (C, C-6), 111.35 (CH, C7), 123.98 (CH, C-8), 120.50 (CH, C-9), 137.-34 (C, C-10), $42.21(\mathrm{CH}, \mathrm{C}-11),-55.75\left(\mathrm{OCH}_{3}, \mathrm{C}-12\right) \mathrm{ppm} ; \mathrm{Ms}: \mathrm{m} / z=360$ $(\mathrm{M})^{+}$. Anal. calcd. (\%) for $\mathrm{C}_{20} \mathrm{H}_{28} \mathrm{~N}_{2} \mathrm{O}_{4}$ : C 62.87, $\mathrm{H} 7.37, \mathrm{~N}$ 9.75; Found (\%): C 62.79, H 7.42,N 9.67\%.

\section{RESULTS AND DISCUSSION}

In this paper, we described the methods for the preparation of new dioximes (DASOX) and diamines derivatives with different bisaldehydes, which have been characterized by spectral data and elemental analysis. Bisaldehydes have been used previously as precursors for macrocyclic-ligand syntheses since they can often be reduced to diamines. The synthetic procedures of newly compounds are shown in Schemes I-III. First, bisaldehyde (1a) was synthesized in good yield by Williamson condensation between 5-bromo-2-hydroxy benzaldehyde and appropriate dibromo propanol in the presence of $\mathrm{NaOH}$ in reflux conditions (Scheme-I). Second, dioximes 2(a-c) was prepared by condensation reaction of hydroxylamine with bisaldehydes $\mathbf{1}(\mathbf{a}-\mathbf{c})$ in the presence of sodium hydroxide which product can be isolated from the ethanol reaction solution by the addition of water. The high yield obtained for this product indicated that side reactions are insignificant under the conditions used for the synthesis (Schemes II and III). Third, the reduction of dioximes 2(a-c) by $\mathrm{LiAlH}_{4}$ in dry THF under nitrogen led to the formation of new diamines 3(a-c) (Schemes II and III). The newly synthesized compounds were characterized by elemental analysis, FT-IR, ${ }^{1} \mathrm{H}$ NMR, ${ }^{13} \mathrm{C}$ NMR and mass spectroscopy.

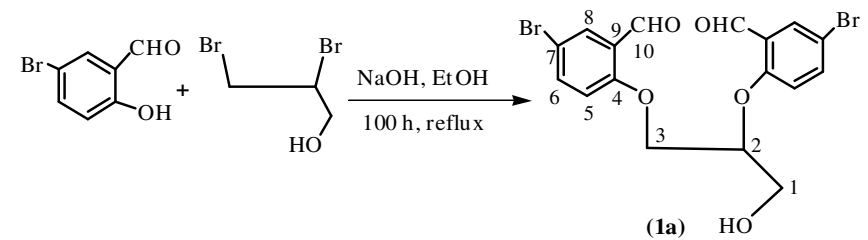

Scheme-I: Synthesis bisaldehyde (1a)<smiles>[X]c1ccc(OCC(CO)COc2ccc([X])cc2C=O)c(C=O)c1</smiles>

(1a), (1b)<smiles>[X]c1ccc(OCC(CO)Oc2ccc([X])cc2CN)c(CN)c1</smiles>

(3a) , (3b)<smiles>[X]c1ccc(OCC(CO)Oc2ccc([X])cc2/C=N/O)c(/C=N/O)c1</smiles>

(2a), (2b)

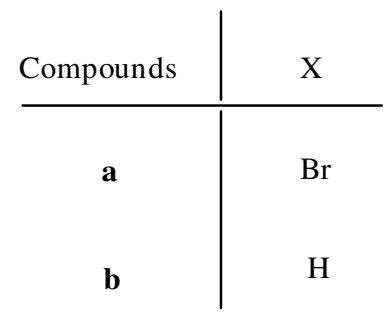<smiles></smiles>

(1c)<smiles>CCO[14CH2]c1ccc(/C=N/O)c(OCCCCCO)c1OC</smiles>

(2c)<smiles>COc1cccc(CN)c1OCCCCCCc1ccc([15NH])c(OC)c1O</smiles>

(3c)

Scheme-III: Synthesis dioxime (2c) and diamine (3c) 
The IR spectra of bisaldehyde 1a revealed a carbonyl mode at $1670 \mathrm{~cm}^{-1}$ and an aromatic mode at $1600-1480 \mathrm{~cm}^{-1}$ and a hydroxyl mode at $3400 \mathrm{~cm}^{-1}$. Absorption band at 686 $\mathrm{cm}^{-1}$ was attributed to the $\mathrm{C}-\mathrm{Br}$ stretching vibration, which also confirm the presence of $\mathrm{Br}$ group. In ${ }^{1} \mathrm{H}$ NMR spectra showed peaks in the region of $\delta$ 4.28-4.34 $\left(\mathrm{OCH}_{2}\right), 4.51-4.55$ $(\mathrm{OCH}), 6.95-7.92(\mathrm{Ar}-\mathrm{H}), 2.8(\mathrm{OH})$ and $10.33(\mathrm{CHO}) .{ }^{13} \mathrm{C}$ NMR spectra values of the carbon atoms at 68.27 (C-1), 114.34 (C-7) and 188.067 (C-10) conformed the formation of bisaldehyde (1a). The NMR spectra are summarized in Table-1 and these spectra are in accord with the proposed structure (Table-1).

\begin{tabular}{cccc}
\multicolumn{4}{c}{ TABLE-1 } \\
\multicolumn{4}{c}{$\begin{array}{c}{ }^{1} \text { HNMR AND }{ }^{13} \text { CNMR SPECTRAL DATA } \\
\text { FOR BISALDEHYDE (1a) }\end{array}$} \\
\hline Atom & $\begin{array}{c}\text { Experimental }^{*} \\
\left({ }^{1} \text { H NMR) }\right.\end{array}$ & Atom & $\begin{array}{c}\text { Experimental }^{*} \\
\left({ }^{13} \text { C NMR) }\right.\end{array}$ \\
\hline H-1,3 & $4.28-4.34(\mathrm{dd})$ & $\mathrm{C}-1$ & 68.27 \\
H-2 & $4.51-4.55(\mathrm{~m})$ & $\mathrm{C}-2$ & 80.7 \\
H-5 & $6.95-6.98(\mathrm{~d})$ & $\mathrm{C}-3$ & 69.67 \\
H-6 & $7.65-7.68(\mathrm{dd})$ & $\mathrm{C}-4$ & 159.12 \\
H-8 & $7.91-7.92(\mathrm{~d})$ & $\mathrm{C}-5$ & 115.04 \\
$\mathrm{H}-10$ & $10.23(\mathrm{~s})$ & $\mathrm{C}-6$ & 138.47 \\
$\mathrm{OH}$ & 2.8 & $\mathrm{C}-7$ & 114.34 \\
& & $\mathrm{C}-8$ & 132.62 \\
& & $\mathrm{C}-9$ & 126.37 \\
& & $\mathrm{C}-10$ & 188.067 \\
\hline
\end{tabular}

*All resonances in ppm downfield from TMS. $\mathrm{CDCl}_{3}$ was used as solvent.

IR spectra of dioximes $\mathbf{2}(\mathbf{a}-\mathbf{c})$ confirm that reaction has occurred by the absence of carbonyl absorption band and the presence of $\mathrm{C}=\mathrm{N}$ and aromatic stretching modes at approximately 1635 and $1600 \mathrm{~cm}^{-1}$. Absorption band at 3400 and 702 $\mathrm{cm}^{-1}$ are characteristic of $\mathrm{OH}$ bonded and $\mathrm{Br}$ group respectively. Aromatic absorption in region $1600-1477 \mathrm{~cm}^{-1}$ was also observed. In the ${ }^{1} \mathrm{H}$ NMR spectra of these compounds $\mathrm{OH}$ and $\mathrm{NOH}$ protons were observed at $\delta 5.6$ and $11.45 \mathrm{ppm}$, respectively. ${ }^{13} \mathrm{C}$ NMR spectra of dioximes 2 (a-c) showed peaks in the region of $\delta-67.68$ (C-1), 112.92 (C-7), 148 (C-10,11) and 110-160 (Ar) ppm, which are in good agreement with the probable structures. The NMR spectral data of the dioxime 2a are summarized in Table- 2 .

TABLE-2

${ }^{1} \mathrm{H}$ NMR AND ${ }^{13} \mathrm{C}$ NMR SPECTRAL DATA FOR DIOXIME (2a)

\begin{tabular}{cccc}
\hline Atom & $\begin{array}{c}\text { Experimental }^{*} \\
\left({ }^{1} \mathrm{H} \mathrm{NMR}\right)\end{array}$ & Atom & $\begin{array}{c}\text { Experimental }^{*} \\
\left({ }^{13} \mathrm{C} \mathrm{NMR}\right)\end{array}$ \\
\hline $\mathrm{H}-1$ & $4.06-4.10(\mathrm{dd})$ & $\mathrm{C}-1$ & 67.68 \\
$\mathrm{H}-2$ & $4.22-4.23(\mathrm{~m})$ & $\mathrm{C}-2$ & 80.7 \\
$\mathrm{H}-3$ & $4.14-4.17(\mathrm{dd})$ & $\mathrm{C}-3$ & 70.58 \\
$\mathrm{H}-5$ & $7.09-7.11(\mathrm{~d})$ & $\mathrm{C}-4$ & 155.75 \\
$\mathrm{H}-6$ & $7.50-7.53(\mathrm{dd})$ & $\mathrm{C}-5$ & 115.71 \\
$\mathrm{H}-8$ & $7.74-7.75(\mathrm{~d})$ & $\mathrm{C}-6$ & 133.38 \\
$\mathrm{H}-10$ & $8.34(\mathrm{~s})$ & $\mathrm{C}-7$ & 112.92 \\
$\mathrm{NOH}$ & 11.45 & $\mathrm{C}-8$ & 127.78 \\
$\mathrm{OH}$ & 5.6 & $\mathrm{C}-9$ & 124.01 \\
& & $\mathrm{C}-10$ & 142.98 \\
\hline
\end{tabular}

*All resonances in ppm downfield from TMS. DMSO was used as solvent.
The IR spectra are typical of aromatic diamines 3(a-c) with major bands at about 1600 and $3400 \mathrm{~cm}^{-1}$ for aromatic rings and $\mathrm{N}-\mathrm{H}, \mathrm{OH}$ stretching modes that are covered each other. In the ${ }^{1} \mathrm{H}$ NMR spectra of diamines $3(\mathbf{a}-\mathbf{c})$, protons $\mathrm{OH}$, $\mathrm{NH}_{2}, \mathrm{OCH}_{3}$ and $\mathrm{Ar}-\mathrm{H}$ appear in theregion 2.7, 3.75-4.8, 3.85 and 6.5-7.3 ppm, respectively. Finally, ${ }^{13} \mathrm{C}$ NMR spectra of these compounds showed peaks at 67.3 (C-1), 41.06-37.2 (C10), $55.75\left(\mathrm{OCH}_{3}\right)$ and 110-157 (Ar) ppm, which corroborate the diamines 3(a-c) character deduced from ${ }^{1} \mathrm{H}$ NMR data. The NMR spectral data of the diamines $\mathbf{3 b}$ are summarized in Table-3.

TABLE-3

${ }^{1} \mathrm{H}$ NMR AND ${ }^{13} \mathrm{C}$ NMR SPECTRAL DATA FOR DIAMINE (3b)

\begin{tabular}{cccc}
\hline Atom & $\begin{array}{c}\text { Experimental } \\
\left({ }^{1} \mathrm{H} \mathrm{NMR}\right)\end{array}$ & Atom & $\begin{array}{c}\text { Experimental }^{*} \\
\left({ }^{13} \mathrm{C} \mathrm{NMR}\right)\end{array}$ \\
\hline $\mathrm{H}-3$ & $4.10-4.13(\mathrm{dd})$ & $\mathrm{C}-1$ & 67.17 \\
$\mathrm{H}-2$ & $4.17-4.24(\mathrm{~m})$ & $\mathrm{C}-2$ & 69.38 \\
$\mathrm{H}-1$ & $4.04-4.07(\mathrm{~m})$ & $\mathrm{C}-3$ & 68.52 \\
$\mathrm{H}-8,6,5,7$ & $6.69-7.18(\mathrm{~m})$ & $\mathrm{C}-4$ & 156.12 \\
$\mathrm{H}-10$ & $3.75(\mathrm{~s})$ & $\mathrm{C}-5$ & 111.78 \\
$\mathrm{NH} 2$ & 4.75 & $\mathrm{C}-6$ & 127.85 \\
$\mathrm{OH}$ & 2.7 & $\mathrm{C}-7$ & 120.18 \\
& & $\mathrm{C}-8$ & 128.50 \\
& & $\mathrm{C}-9$ & 128.95 \\
& & $\mathrm{C}-10$ & 41.06 \\
\hline
\end{tabular}

*All resonances in ppm downfield from TMS. $\mathrm{CDCl}_{3}$ was used as solvent.

Mass spectral fragments of all newly synthesized compounds agree with the proposed structure. The elemental analysis of these compounds showed good agreements with calculated values of \% C, $\mathrm{H}$ and $\mathrm{N}$.

\section{Conclusion}

In this article, an efficient, convenient method is generated for the synthesis of new dioximes and diamines in high yield under reflux conditions. This method may be used to prepare various amine compounds which have a wide applications in organic synthesis.

\section{ACKNOWLEDGEMENTS}

The authors acknowledge to Payame Noor University, Ardabil, Iran for providing facilities for this work.

\section{REFERENCES}

1. A. Para, Carbohydr. Polym., 57, 277 (2004); https://doi.org/10.1016/j.carbpol.2004.05.005.

2. G.L. Kad, M. Bhandari, J. Kaur, R. Rathee and J. Singh, Green Chem., 3, 275 (2001); https://doi.org/10.1039/b107356g

3. A.F. Abdel-Magid, K.G. Carson, B.D. Harris, C.A. Maryanoff and R.D. Shah, J. Org. Chem., 61, 3849 (1996);

https://doi.org/10.1021/jo960057x.

4. K. Abiraj and D.C. Gowda, J. Chem. Res., 2003, 332 (2003); https://doi.org/10.3184/030823403103174281.

5. A.F. Abdel-Magid and S.J. Mehrman, Org. Process Res. Dev., 10, 971 (2006);

https://doi.org/10.1021/op0601013.

6. D.D. Perrin and W.L.F. Armarego, Purification of Laboratory Chemicals, Pergamon Press, Oxford, edn 3 (1988).

7. L.G. Armstrong and I.F. Lindoy, Inorg. Chem., 14, 1322 (1975); https://doi.org/10.1021/ic50148a024. 